\title{
STX1A and Asperger syndrome: a replication study
}

\author{
Jaroslava Durdiaková ${ }^{1}$, Varun Warrier ${ }^{1}$, Sharmila Banerjee-Basu ${ }^{4}$, Simon Baron-Cohen ${ }^{1,2^{*}}$ \\ and Bhismadev Chakrabarti ${ }^{1,3^{*}}$
}

\begin{abstract}
Background: Autism spectrum conditions (ASC) are a group of conditions characterized by difficulties in communication and social interaction, alongside unusually narrow interests and repetitive, stereotyped behaviour. Genetic association and expression studies have suggested an important role for the GABAergic circuits in ASC. Syntaxin 1A (STX1A) encodes a protein involved in regulation of serotonergic and GABAergic systems and its expression is altered in autism.
\end{abstract}

Methods: In this study, the association between three single nucleotide polymorphisms (SNPs) (rs4717806, rs941298 and rs6951030) in STX1A gene and Asperger syndrome (AS) were tested in 650 controls and 479 individuals with AS, all of Caucasian ancestry.

Results: rs4717806 ( $P=0.00334)$ and rs941298 $(P=0.01741)$ showed a significant association with AS, replicating previous results. Both SNPs putatively alter transcription factor binding sites both directly and through other variants in high linkage disequilibrium.

Conclusions: The current study confirms the role of STXIA as an important candidate gene in ASC. The exact molecular mechanisms through which STXIA contributes to the etiology remain to be elucidated.

Keywords: Asperger syndrome, Serotonergic system, Single nucleotide polymorphism, STXIA

\section{Background}

Autism spectrum conditions (ASC) are characterized by difficulties in social interaction and impaired communication, alongside unusually narrow interests and repetitive, stereotyped behaviour (DSM-IV). Asperger syndrome (AS), a subcategory of ASC, differs from other autism conditions by lack of any delay in language and cognitive development [1]. ASC has a prevalence of 1 in 100 [2] and heritability of around 80\% [3]. Several genes involved in neural transmission, and in particular GABAergic neurotransmission, have been implicated in ASC [4-7].

Syntaxin 1A (STX1A) is an important component of the GABAergic system, due to its involvement in the transport of glutamate and its conversion to gamma-aminobutyric

\footnotetext{
* Correspondence: sb205@cam.ac.uk; b.chakrabarti@reading.ac.uk 'Autism Research Centre, Department of Psychiatry, University of Cambridge, 18b Trumpington Road, Cambridge CB2 8AH, UK

${ }^{3}$ Centre for Integrative Neuroscience and Neurodynamics, School of Psychology and Clinical Language Sciences, University of Reading, Reading RG6 6AL, UK
} Full list of author information is available at the end of the article acid (GABA) [8]. The protein STX1A is expressed presynaptically and is involved in neurotransmitter release and synaptic vesicle docking and thus is an essential factor for neurotransmission. It also interacts with serotonin and alters its subcellular localization and expression [9]. Atypical function in both serotonergic and GABAergic neurotransmission systems has been reported in autism [10]. GABAergic signaling during embryonic development contributes to the excitatory/inhibitory signaling balance in neural networks. Dysregulation of GABAergic system during development is thought to account for some of the sensory sensitivity in people with ASC [6].

The gene STX1A consists of 10 exons (Figure 1) spanning a $20.42 \mathrm{~kb}$ region (http://www.genome.ucsc.edu). It lies on 7q11.23, a locus that has been reported to be duplicated in some individuals with autism, and associated with deletions in Williams syndrome [11]. A possible role for STX1A in the development of autism has previously been suggested. Three single nucleotide polymorphisms (SNPs) (rs2293485 and rs4717806 in the Caucasian population, 


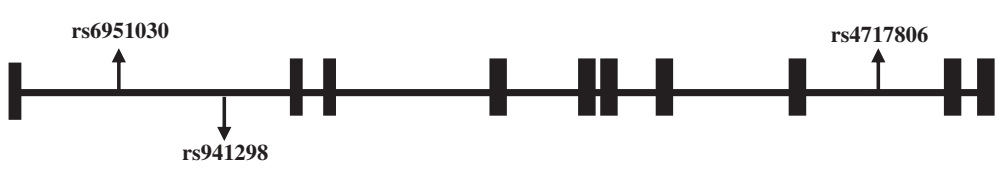

Figure 1 Genomic structure of STX1A and the location of tested SNPs. Exons are indicated by boxes; introns are indicated by lines; SNP positions are denoted by arrows (http://www.genome.ucsc.edu).

and rs69510130 in the Japanese population) are nominally associated with high functioning autism (HFA) [12,13]. rs4717806 has also been related to early developmental differences in people with autism [12]. Another SNP, rs1569061, is nominally associated with ASC in a Caucasian population [11]. Additionally, STX1A mRNA expression is higher in lymphocytes of people with HFA [12], and lower in the anterior cingulated gyrus in post-mortem brain tissue [13], compared to matched controls. This makes STX1A an interesting candidate gene for ASC. In this study, we examined the association of three SNPs in STX1A with AS, in a Caucasian sample, in an effort to replicate previous findings.

\section{Methods}

All individuals enrolled in the current study were adults of Caucasian origin from the same geographic region (the United Kingdom). Participants were asked to complete an online version of the Autism Spectrum Quotient (AQ) test [14], which is a measure of autistic traits. A score of 32 and above is an excellent predictor of ASC [15], and the mean $\mathrm{AQ}$ score in the general population is $16.4(\mathrm{SD}=$ 6.3) [14]. Individuals with a clinical diagnosis of AS ( $\mathrm{n}=$ 479; 97 females, 382 males) took part and achieved a mean AQ score of $35.8 \pm 8.3$. The control group comprised participants ( $\mathrm{n}=650 ; 367$ females, 284 males) chosen to have an AQ score below 24, to ensure a balanced representation of individuals from both ends of the autistic trait continuum. The mean AQ score within the control population was $14.8 \pm 5.2$. Consent was obtained from all the participants. This study was approved by the University of Cambridge Psychology Research Ethics Committee and the NHS National Research Ethics Service.

Based on previously published data, three SNPs (rs4717806, rs941298, rs6951030) were selected and analyzed in this study. rs4717806 and rs69510130 have been reported to be nominally associated with HFA in the Caucasian and Japanese population, respectively $[12,13]$. The third SNP, rs941298, is in high linkage disequilibrium (LD) with rs4717806 $\left(R^{2}=0.96\right)$ and in moderate LD with an exonic SNP, rs2293485 $\left(R^{2}=0.67\right)$, which is nominally associated with HFA in the Caucasian population [12]. LD values between SNPs of interest in the HapMap CEPH European samples of the Utah Residents with Northern and Western European Ancestry (CEU) population data were calculated using SNAP (http://www. broadinstitute.org/mpg/snap/). The minor allele frequency (MAF) for the tested SNPs was above 0.05 in the CEPH CEU population as calculated from the dbSNP database (http://www.ncbi.nlm.nih.gov/projects/SNP/). LD plots for the sample studied were created using Haploview [16].

DNA was extracted from buccal swabs and anonymized. SNP genotyping was performed using TaqMan SNP Genotyping Assays (Applied Biosystems Inc., CA, USA) according the previously described protocol [17]. Allelic association testing was performed using Plink v1.07 (http:// pngu.mgh.harvard.edu/ purcell/plink/) [18]. Bonferroni correction was performed to correct for multiple SNPs tested after evaluating the number of completely independent SNPs and determination of a new alpha value using SNPSpD (http://gump.qimr.edu.au/general/daleN/ SNPSpD/) [19]. This was done as Bonferroni correction is over-corrective when the SNPs are in LD. $P$ values were reported as significant if they were below the alpha threshold.

Functional annotation was performed using Haploreg (http://www.broadinstitute.org/mammals/haploreg/haploreg. php) [20], SNPnexus (http://snp-nexus.org/) [21], and FSNP (http://compbio.cs.queensu.ca/F-SNP/) [22].

\section{Results}

In the association analyses, rs4717806 $(P=0.003)$ and rs941298 $(P=0.017)$ showed significant association with AS. These results remained significant after correcting for multiple testing. The estimated average number of independent loci was 2 . The SNPSpD determined threshold $P$ value was 0.023 . None of the SNPs deviated from the Hardy-Weinberg equilibrium at $P<0.05$. Genotyping rate was above $98 \%$. The test statistics are summarized in Table 1. In our sample, all three SNPs are part of a common LD block as calculated by Haploview (Figure 2).

\section{Discussion}

In this study, three SNPs in STX1A were tested for association with AS to replicate findings previously reported in the literature. We found that two of these SNPs (rs 4717806 and rs941298) were significantly associated with a clinical diagnosis of AS. The odds ratio for the two significant SNPs was below 0, indicating the protective role of the minor allele. rs4717806 and rs69510130 have been reported to be nominally associated with HFA in previous reports $[12,13]$. The direction of effect is in agreement 
Table 1 Single SNP association analyses

\begin{tabular}{lllllllll}
\hline dbSNp ID & Alleles $^{\mathbf{a}}$ & MAF $^{\mathbf{b}}$ & F_A $^{\mathbf{c}}$ & F_U $^{\mathbf{d}}$ & CHISQ $^{\mathbf{e}}$ & Odds ratio & $\boldsymbol{P}_{\text {value }}$ & Alpha $^{\mathbf{9}}$ \\
\hline rs6951030 & T/G & 0.18 & 0.19 & 0.17 & 2.40 & 1.24 & 0.06 & 0.023 \\
rs4717806 & T/A & 0.33 & 0.29 & 0.36 & 9.49 & 0.76 & 0.003 & 0.023 \\
rs941298 & G/A & 0.33 & 0.29 & 0.35 & 6.09 & 0.80 & $\mathbf{0 . 0 1 7}$ & 0.023 \\
\hline
\end{tabular}

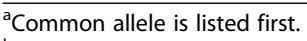

${ }^{\mathrm{b} C a l c u l a t e d}$ by Plink v1.07 in analysed sample.

${ }^{\mathrm{c}}$ The frequency of this variant in cases.

${ }^{\mathrm{d}}$ The frequency of this variant in controls.

${ }^{\mathrm{e}}$ The $\mathrm{X}^{2}$ statistic for this test ( $1 \mathrm{df}$ ).

${ }^{f}$ Computed on the basis of likelihood ratio test.

${ }^{9}$ Determined after evaluating the number of completely independent SNPs using SNPSpD.

with our findings. Both SNPs have been under-transmitted from non-affected parents to affected probands. rs 941298 is in high LD with rs4717806 $\left(R^{2}=0.96\right)$ and in moderate LD with exonic rs2293485 $\left(R^{2}=0.67\right)$, which is also nominally associated with HFA [12].

We identified significant association between rs4717806 and AS, supporting previously published data [12]. rs941298 was also significantly associated with AS in the

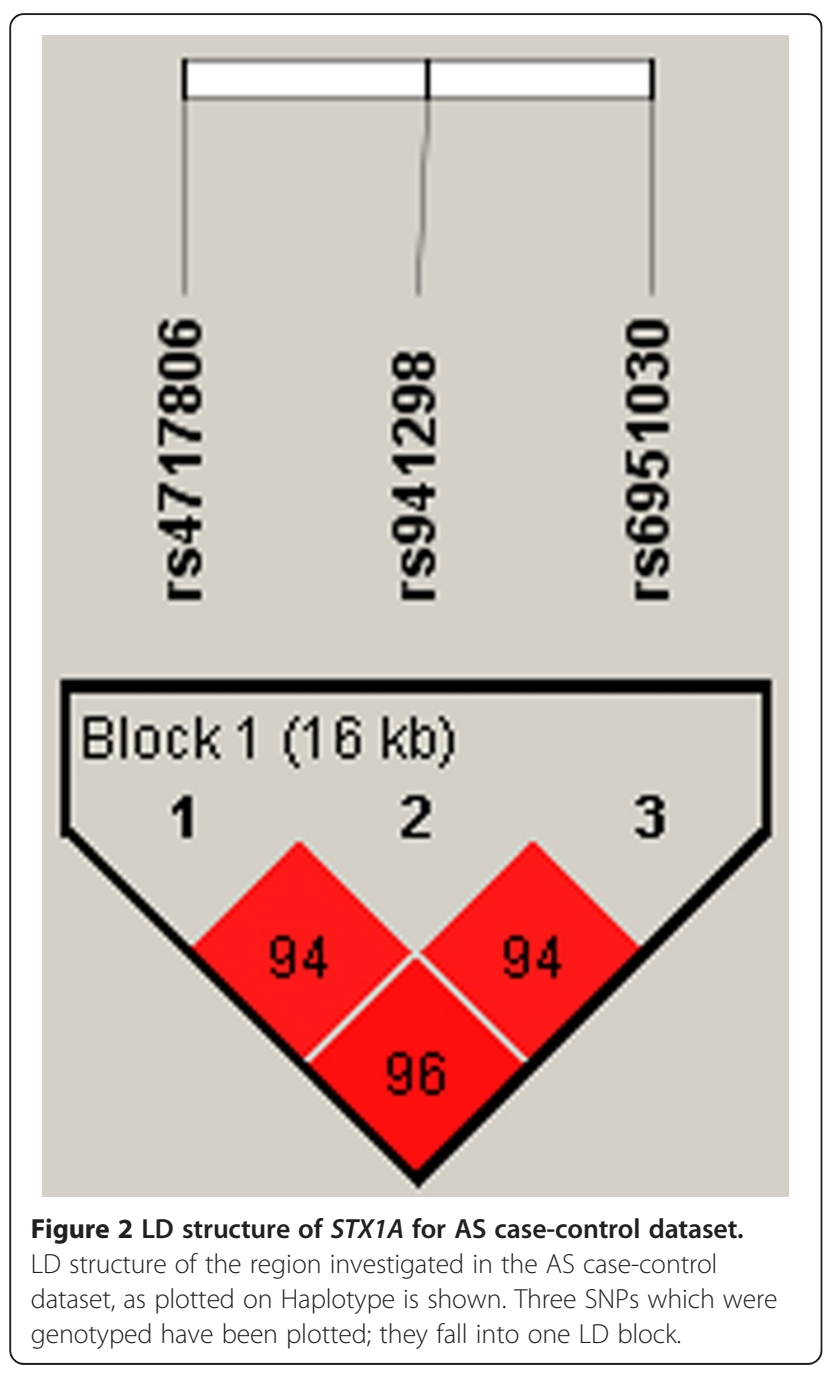

current study. We were not able to replicate the nominal association between rs69510130 and HFA [13] in our sample. However, rs69510130 was nominally significant in a Japanese sample in the previous study, whereas we tested for association in a Caucasian sample.

Both SNPs (rs4717806, rs941298) that showed significant association with AS in our study are intronic SNPs. rs4717806 lies in intron 9 and rs941298 lies in intron 2; they are 8,786 bp apart, in high LD in our sample $\left(R^{2}=\right.$ 0.92), and part of a common LD block as calculated by Haploview (Figure 2). Both the SNPs alter various transcription binding sites both directly and through other SNPs in LD (http://www.broadinstitute.org/mammals/ haploreg/). There are no copy number polymorphisms or miRNA binding sites associated with either of the SNPs (http://www.snp-nexus.org/). rs4717806 is highly conserved across species according to F-SNP. It is likely that the expression of STXA1 is regulated by these SNPs along with additional cis- and trans-acting factors.

While this study replicates the results from previous studies [12,13], it is different in that we focused on AS, rather than HFA or classic autism. Further, the previous studies used Transmission Disequilibrium Tests to identify over-transmitted alleles in family trios of individuals with ASC and HFA [12]. The current study design is population based and with a moderate sample size; hence, these results need to be replicated in a larger sample. It is worth mentioning that females are overrepresented in the control sample compared to the AS group. Men typically score higher than women in the self-reported AQ test [14], which may account for the lower AQ score describing the control population. In the sex-stratified analyses, none of the SNPs are significant after Bonferroni correction indicating that the over-representation of females in controls is not driving the association (data not shown).

Additionally, only three SNPs were tested, chosen specifically to replicate previous results. The high LD between rs4717806 and rs941298 must be taken into account when interpreting the results. It is likely that both the SNPs are tagging one single causative locus, which is reflected in the significant $P$ values of the two SNPs. rs941298 would not remain significant if traditional Bonferroni correction 
was applied. A single gene is unlikely to have a major effect in complex conditions like autism, and many other genes are likely to contribute to the phenotype. This study, in combination with previous studies, provides evidence for STX1A as involved in the etiology of ASC. Further cellular, molecular, and neurocognitive studies are required to elucidate the role of this gene in ASC.

\section{Conclusions}

rs4717806 $(P=0.00334)$ and rs941298 $(P=0.01741)$ showed significant association with AS, replicating findings in previous studies in the same direction. Both SNPs putatively alter transcription factor binding sites both directly and through other variants in high linkage disequilibrium. The current study confirms the role of STX1A as an important candidate gene in ASC. The exact molecular mechanisms through which STX1A contributes to the etiology remain to be elucidated.

\section{Abbreviations}

AQ: Autism spectrum quotient; AS: Asperger syndrome; ASC: Autism spectrum conditions; CEU: European samples of Utah Residents with Northern and Western European Ancestry, GABA, Gamma-aminobutyric acid; HFA: High-functioning autism; LD: Linkage disequilibrium; MAF: Minor allele frequency; SNP: Single nucleotide polymorphism; STX1A: Syntaxin 1A.

\section{Competing interests}

The authors declare that they have no competing interests.

\section{Authors' contributions}

BC and SBC co-designed the study. SBC obtained funding for the study. SBB suggested the mapping of STXIA as a relevant candidate gene. WW and JD conducted the analysis. JD wrote the first draft of the paper revised by all authors. All authors read and approved the final manuscript.

\section{Acknowledgements}

This study was funded by grants to SBC by Target Autism Genome, the Autism Research Trust, the MRC UK, and the Max Planck Institute for Psycholinguistics, Nijmegen. We are grateful to Jonathan Breidbord, Allen Chan, Agnese Di Napoli, Laura Murphy, and Simon Fisher for help at various stages of the project.

\section{Author details \\ 'Autism Research Centre, Department of Psychiatry, University of Cambridge, 18b Trumpington Road, Cambridge CB2 8AH, UK. ${ }^{2}$ CLASS Clinic, Cambridgeshire and Peterborough NHS Foundation Trust (CPFT), Fulbourn, Cambridge CB21 5EF, UK. ${ }^{3}$ Centre for Integrative Neuroscience and Neurodynamics, School of Psychology and Clinical Language Sciences, University of Reading, Reading RG6 6AL, UK. ${ }^{4}$ Mindspec Inc, 9656 Blake Ln, Fairfax, VA 22031, USA.}

Received: 11 December 2013 Accepted: 5 February 2014 Published: 18 February 2014

\section{References}

1. McPartland J, Klin A: Asperger's syndrome. Adolesc Med Clin 2006, 17(3):771-788.

2. Baron-Cohen S, Scott FJ, Allison C, Williams J, Bolton P, Matthews FE, Brayne C: Prevalence of autism-spectrum conditions: UK school-based population study. Br J Psychiatry 2009, 194(6):500-509.

3. Klei L, Sanders SJ, Murtha MT, Hus V, Lowe JK, Willsey AJ, Moreno-De-Luca D, Yu TW, Fombonne E, Geschwind D, Grice DE, Ledbetter DH, Lord C, Mane SM, Martin CL, Martin DM, Morrow EM, Walsh CA, Melhem NM, Chaste P, Sutcliffe JS, State MW, Cook EH Jr, Roeder K, Devlin B: Common genetic variants, acting additively, are a major source of risk for autism. Mol Autism 2012, 3(1):9.
4. Buxbaum JD, Silverman JM, Smith CJ, Greenberg DA, Kilifarski M, Reichert J, Cook EH Jr, Fang Y, Song CY, Vitale R: Association between a GABRB3 polymorphism and autism. Mol Psychiatry 2002, 7(3):311-316.

5. Fatemi SH, Reutiman TJ, Folsom TD, Thuras PD: GABA(A) receptor downregulation in brains of subjects with autism. J Autism Dev Disord 2009, 39(2):223-230.

6. Pizzarelli R, Cherubini E: Alterations of GABAergic signaling in autism spectrum disorders. Neural Plast 2011, 2011:297153.

7. Tavassoli T, Auyeung B, Murphy LC, Baron-Cohen S, Chakrabarti B: Variation in the autism candidate gene GABRB3 modulates tactile sensitivity in typically developing children. Mol Autism 2012, 3(1):6.

8. Yu YX, Shen L, Xia P, Tang YW, Bao L, Pei G: Syntaxin 1A promotes the endocytic sorting of EAAC1 leading to inhibition of glutamate transport. J Cell Sci 2006, 119(Pt 18):3776-3787.

9. Haase J, Killian AM, Magnani F, Williams C: Regulation of the serotonin transporter by interacting proteins. Biochem Soc Trans 2001, 29(Pt 6):722-728.

10. Coghlan S, Horder J, Inkster B, Mendez MA, Murphy DG, Nutt DJ: GABA system dysfunction in autism and related disorders: from synapse to symptoms. Neurosci Biobehav Rev 2012, 36(9):2044-2055.

11. Malenfant P, Liu X, Hudson ML, Qiao Y, Hrynchak M, Riendeau N, Hildebrand MJ, Cohen IL, Chudley AE, Forster-Gibson C, Mickelson EC, Rajcan-Separovic E, Lewis ME, Holden JJ: Association of GTF2i in the Williams-Beuren syndrome critical region with autism spectrum disorders. J Autism Dev Disord 2012, 42(7):1459-1469.

12. Nakamura K, Anitha A, Yamada K, Tsujii M, Iwayama Y, Hattori E, Toyota T, Suda S, Takei N, Iwata Y, Suzuki K, Matsuzaki H, Kawai M, Sekine Y, Tsuchiya KJ, Sugihara G, Ouchi Y, Sugiyama T, Yoshikawa T, Mori N: Genetic and expression analyses reveal elevated expression of syntaxin 1A (STX1A) in high functioning autism. Int I Neuropsychopharmacol 2008, 11(8):1073-1084.

13. Nakamura K, Iwata Y, Anitha A, Miyachi T, Toyota T, Yamada S, Tsujii M, Tsuchiya K, Iwayama Y, Yamada K, Hattori E, Matsuzaki H, Matsumoto K, Suzuki K, Suda S, Takebayashi K, Takei N, Ichikawa H, Sugiyama T, Yoshikawa $T$, Mori N: Replication study of Japanese cohorts supports the role of STX1A in autism susceptibility. Prog Neuropsychopharmacol Biol Psychiatry 2011, 35(2):454-458.

14. Baron-Cohen S, Wheelwright S, Skinner R, Martin J, Clubley E: The autismspectrum quotient (AQ): evidence from Asperger syndrome/highfunctioning autism, males and females, scientists and mathematicians. J Autism Dev Disord 2001, 31(1):5-17.

15. Woodbury-Smith MR, Robinson J, Wheelwright S, Baron-Cohen S: Screening adults for Asperger Syndrome using the AQ: a preliminary study of its diagnostic validity in clinical practice. J Autism Dev Disord 2005, 35(3):331-335.

16. Barrett JC, Fry B, Maller J, Daly MJ: Haploview: analysis and visualization of LD and haplotype maps. Bioinformatics 2005, 21(2):263-265.

17. Chakrabarti B, Dudbridge F, Kent L, Wheelwright S, Hill-Cawthorne G, Allison C, Banerjee-Basu S, Baron-Cohen S: Genes related to sex steroids, neural growth, and social-emotional behavior are associated with autistic traits, empathy, and Asperger syndrome. Autism Res 2009, 2(3):157-177.

18. Purcell $S$, Neale B, Todd-Brown K, Thomas L, Ferreira MA, Bender D, Maller J, Sklar P, de Bakker PI, Daly MJ, Sham PC: PLINK: a tool set for wholegenome association and population-based linkage analyses. Am J Hum Genet 2007, 81(3):559-575.

19. Nyholt DR: A simple correction for multiple testing for single-nucleotide polymorphisms in linkage disequilibrium with each other. Am J Hum Genet 2004, 74(4):765-769.

20. Ward LD, Kellis M: HaploReg: a resource for exploring chromatin states, conservation, and regulatory motif alterations within sets of genetically linked variants. Nucleic Acids Res 2012, 40(Database issue):D930-934.

21. Chelala C, Khan A, Lemoine NR: SNPnexus: a web database for functional annotation of newly discovered and public domain single nucleotide polymorphisms. Bioinformatics 2009, 25(5):655-661.

22. Lee PH, Shatkay H: An integrative scoring system for ranking SNPs by their potential deleterious effects. Bioinformatics 2009, 25(8):1048-1055.

doi:10.1186/2040-2392-5-14

Cite this article as: Durdiaková et al: STX1A and Asperger syndrome: a replication study. Molecular Autism 2014 5:14. 